\title{
Very large optical-infrared telescopes: today and tomorrow
}

\author{
Malcolm S. Longair \\ Cavendish Laboratory, University of Cambridge, JJ Thomson Avenue, Cambridge CB3 0HE, \\ England, UK.
}

\begin{abstract}
Some of the achievements and capabilities of the current generation of 8-10 metre optical-infrared telescopes are reviewed. The challenges of building yet larger optical-infrared telescopes in the diameter range 30 to 100 metres are discussed.
\end{abstract}

Keywords. Telescopes, History of astronomy, Future prospects.

\section{Dedication}

This lecture is dedicated to the memory of Dr. Robert (Bob) Stobie. Bob was one of my oldest friends and colleagues. We started our PhDs in Cambridge in the same year 1963, we shared a flat in our second year of research, I was an usher at his wedding, we played music together and he was my Deputy Director when I was Director of the Royal Observatory Edinburgh. It is a great sadness that he died in 2002 before the results of his enormous efforts, as Director of the South African Astronomical Observatory, to bring about the creation of the Southern African Large Telescope could be celebrated.

\section{Introduction}

In this brief review, I discuss the following topics. Firstly, the consequences of the enormous increase in observing capability in the optical-infrared waveband of the last 10-15 years are reviewed. Then, I present examples of the achievements and capabilities of the present generation of 8-10 metres telescopes and set them in the context of the achievements of other telescopes for the optical-infrared waveband and for other wavebands. Next, I review the changing perspective of astronomers in the era of very large telescopes and outline my views on the challenges involved in building telescopes in the 30 to 100 metre diameter range. Finally, I consider the issue of winning over the hearts and minds of astronomers and astrophysicists for the support of these very large projects.

\section{The explosion of observing capability}

The enormous increase in observing capability over recent years is most dramatically illustrated by a diagram kindly provided by Dr. Matt Mountain of the total amount of telescope collecting area available to astronomers as a function of time over the last 400 years (Figure 1). The increase in collecting area over the last 15 years is extraordinary, the various 8-10 metre facilities responsible for the rise being indicated in the inset. Discontinuities are associated with the commissioning of the two Keck telescopes, the four telescopes of the VLT, Gemini, Subaru and the HET telescopes. Thus, the era of the 8-10 metres telescopes is very recent and it is too soon to make any serious attempt at assessing their impact upon astrophysics and cosmology. It is much easier to provide an 


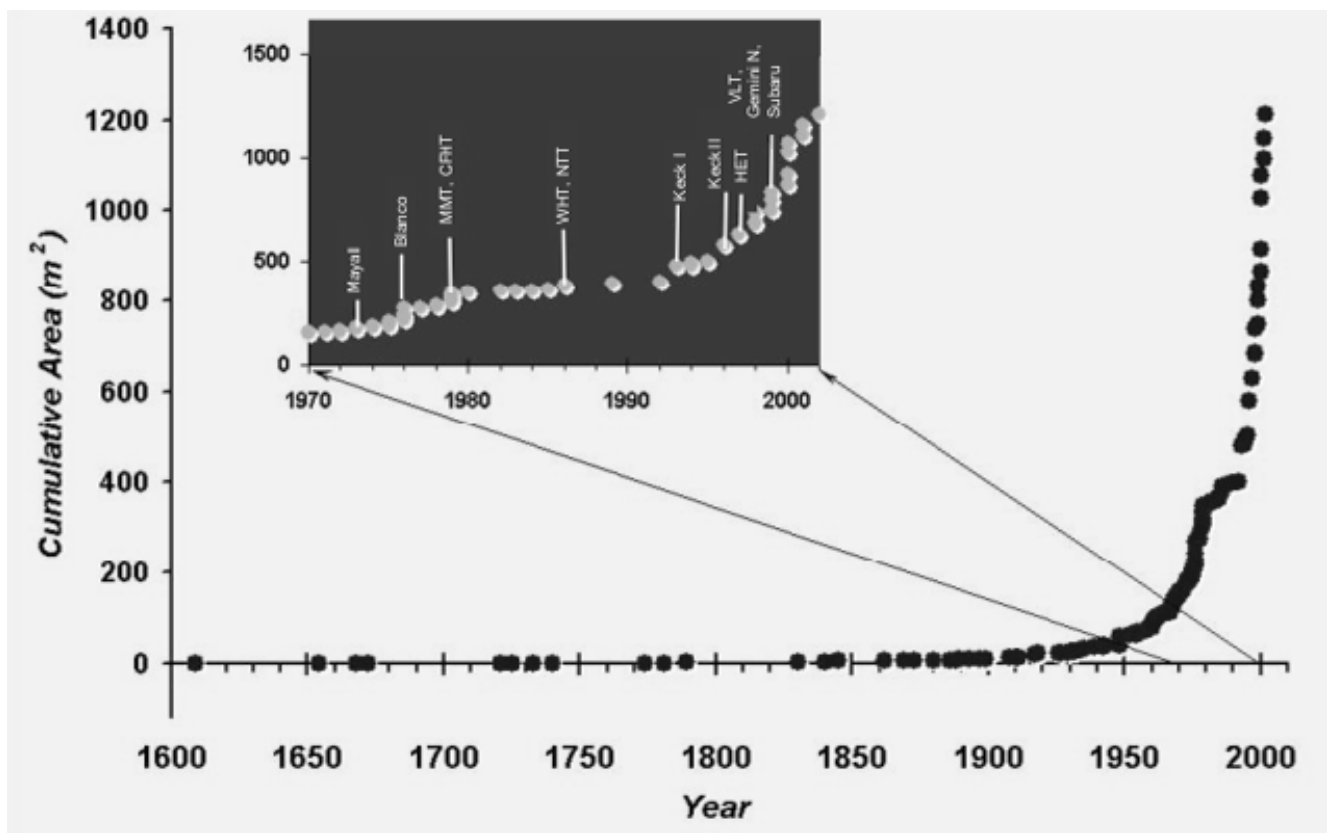

Figure 1. The cumulative collecting area of optical-infrared telescopes available to astronomers from the time of Galileo to 2005. The inset shows the time interval 1970 to 2005 with the date of commissioning of various large facilities indicated. (Courtesy of Dr. Matt Mountain).

assessment of their remarkable demonstrated capabilities. Many topics which astronomers of the 1960s would have regarded as the purest wishful thinking are now the bread and butter of the present generation of astronomers.

At the same time, we need to be aware of the crucial complementarity with other optical-infrared telescopes over recent years. Prime among these are the extraordinary achievements of the Hubble Space Telescope, as well as the key science coming from the Sloan Digital Sky Survey, the Two-Degree Field of the Anglo-Australian Telescope and so on. Indeed, these telescope facilities have had a huge impact upon the advance of astrophysics and cosmology during the early years of operation of the 8-10 metres telescopes and make the point that the very large telescopes should be regarded as part of the armoury of the astronomer in tackling astrophysics and cosmology in these wavebands.

In addition, we need to take into account complementarity with achievements in other wavebands. To name only the obvious examples, no assessment of the achievements of modern astronomy would be complete without the science which has come from the Chandra X-ray Observatory, the Newton-XMM X-ray Observatory, the Wilkinson-MAP, the various Supernova projects, the Very Large Array, the Compton Gamma-Ray Observatory and so on. This list reinforces the point that the 8-10 metres telescopes should be regarded as part of a continuum of facilities which span the complete electromagnetic spectrum accessible to astronomers, as well as non-electromagnetic astronomies fostered, for example, by the Gravitational Wave and Neutrino Observatories.

This introduction makes the point that the astrophysics and cosmology of the twentyfirst century have at their core the extension of observational capabilities for all wavebands. This has resulted in new ways of tackling astronomical problems. Many of the new approaches involve extensions of existing capabilities - angular and spectral resolution, sensitivity, the sizes of data-sets, new techniques for astronomy, new concepts in physics, 
astrophysics and cosmology, new regions of parameter space and so on. In addition, we should regard the astrophysics of the 21st century as a partnership between space and ground-based astronomy and technology, including data analysis. Into the mix we need to add the advance of theory, including supercomputing. These considerations lead to:

Lesson 1. We should consider the Extremely Large Optical-Infrared Telescope projects as part of a global strategy involving all other wavebands and approaches. Not only is this an exciting holistic approach to astrophysics and cosmology but, in more practical terms, we will not get the support necessary to afford these telescopes unless the whole astronomical community is behind them.

\section{Examples of the capability of the 8-10 metres telescopes}

It is simplest to carve up astronomy into the four areas which the organisers of this symposium have adopted:

- Exoplanets and star formation

- Stellar populations

- Intergalactic medium and Large scale structures

- Galaxy formation/evolution and cosmology, including Active Galactic Nuclei (AGN).

It is clearly impossible to make an objective assessment of the achievements of the present generation of very large telescopes - I am encouraged that the reviewers of the four areas listed above will do much more expert justice that I can. Consequently, I will adopt what I will call the "Olivier Messiaen approach" to this review. Alexander Goehr has recounted that, in his master classes on composition at the Paris Conservatoire, Messiaen's approach was simply play an excerpt from a piece of music and say, "I like that". I will adopt the same approach. I have taken my examples from the areas with which I am familiar and from press releases over the last two years from the various telescope projects. All these topics are dealt with in much greater detail in the reviews which follow and which include detailed literature references.

\subsection{Exoplanets and star formation}

Undoubtedly, the explosion of activity in the area of exoplanets and of the embryonic discipline of astrobiology has been one of the most remarkable achievements of modern observational astronomy. As of 11 October 2005, when I last looked, there were 169 extra-solar planets in 145 planetary systems, including 18 multiple planet systems. The 8-10 metres telescopes have played their full part in this activity, Figure 2(a) showing a recent determination of the tiny velocity changes in two planetary eclipses as observed by the VLT. Only a relatively small number of exoplanets exhibit such eclipses, but these cases are of special importance because the properties of the planetary atmosphere can be studied, as has already been demonstrated by the Hubble Space Telescope for the exoplanet HD209458. In that system, evidence for sodium in the planetary atmosphere was found, thanks to the superb stability of the STIS spectrograph on board the HST.

Just as we were getting used to the idea that brown dwarfs and exoplanets are now integral parts of observational astrophysics, the first image of a planet orbiting the brown dwarf 2M1207 was announced (Figure 2(b)). Just as exciting as the science itself is the fact that this system was observed using the NACO adaptive-optics facility at the $8.2-\mathrm{m}$ VLT Yepun Telescope.

Another lovely example of the capabilities of the 8-10 metres telescopes for planetary science is the high resolution spectroscopy of the Planet 2003 UB313, which is in a orbit 

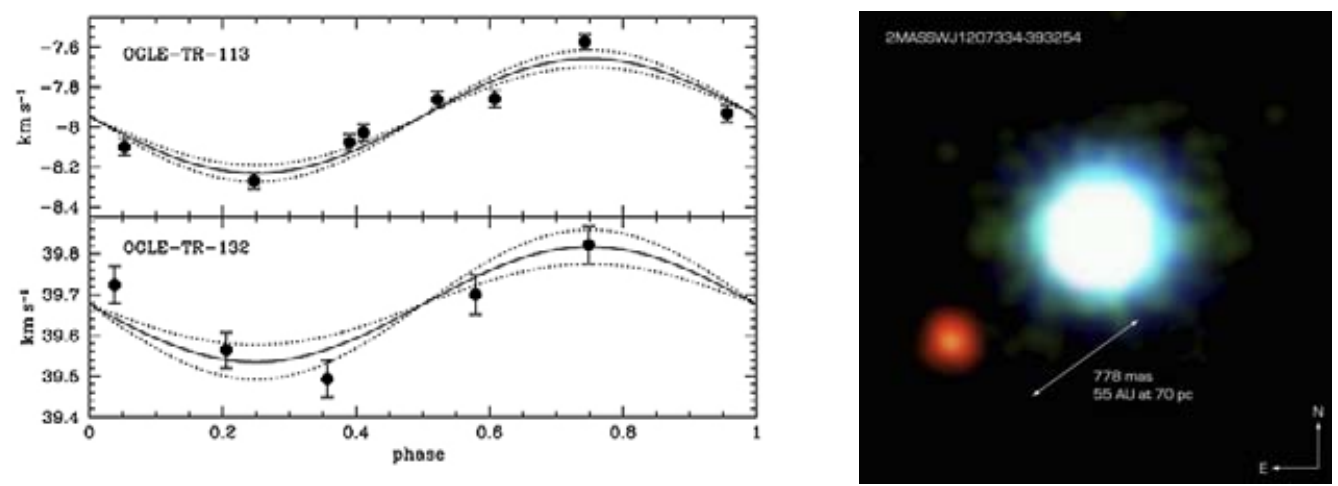

Figure 2. (a) Velocity variations of two stars with transiting exoplanets observed by the VLT KUEYEN with the FLAMES spectrograph (ESO Press Release 11/04). (b) The first planet to be imaged orbiting a brown dwarf using the NACO adaptive-optics facility at the 8.2-m VLT YEPUN (ESO Press Release 12/05).

which normally places it outside the orbit of Pluto. The spectrum shows methane bands, similar to those observed in the spectrum of Pluto.

I am very strongly impressed by the enormous potential for advances in the studies of exoplanets, in all their diversity, with the present and future generation of large telescopes.

\subsection{Stellar populations}

Interpreting the term 'stellar populations' rather generously, the 8-10 metres telescopes have shown their capability in uncovering unexpected aspects of stellar populations and their evolution. A lovely example is the double main sequence observed in the globular cluster $\omega$-Centauri (Figure 3(a)). The lower sequence is interpreted as a helium rich main sequence and must have arisen through the coalescence of the old population with a younger system which had increased helium abundance. In addition to its astrophysical interest, the spectroscopy of the stars in the cluster was undertaken using multi-object spectroscopy, the stars for which spectra could be observed being indicated by the larger symbols. The diagram indicates clearly the magnitude limit of about 21 for multi-object spectroscopy at the resolution needed for these studies, even with one of the VLT telescopes.

Another lovely example of the power of the VLTs, now used in interferometric mode, is the recent measurement of the changes in diameter of the Cepheid variable L Carinae through a complete cycle of its variability using the VINCI interferometric system. Figure 3(b) shows the remarkable capability of a pair of 8-metre telescopes for determining less than $10 \%$ changes in diameter of the Cepheid variable. Besides its intrinsic interest for the astrophysics of pulsating stars, these observations are of great importance in improving the precision with which the Baade-Wesselink method of distance determination can be applied to Cepheid variables. This calibration is one of the key steps in the determination of the cosmic distance scale by the traditional route adopted, for example, by the HST Key Project to measure Hubble's constant $H_{0}$.

Another beautiful example of the power of multi-object spectroscopy for studies of the stellar populations of galaxies is provided by the average spectra of distant galaxies as observed by the VLT. These spectra reveal a wide range of spectral features which enable the stellar populations of these galaxies to be studied directly. It can be seen in Figure 4 that the ranges of redshifts studied now extend to redshifts as large as 5 . While 

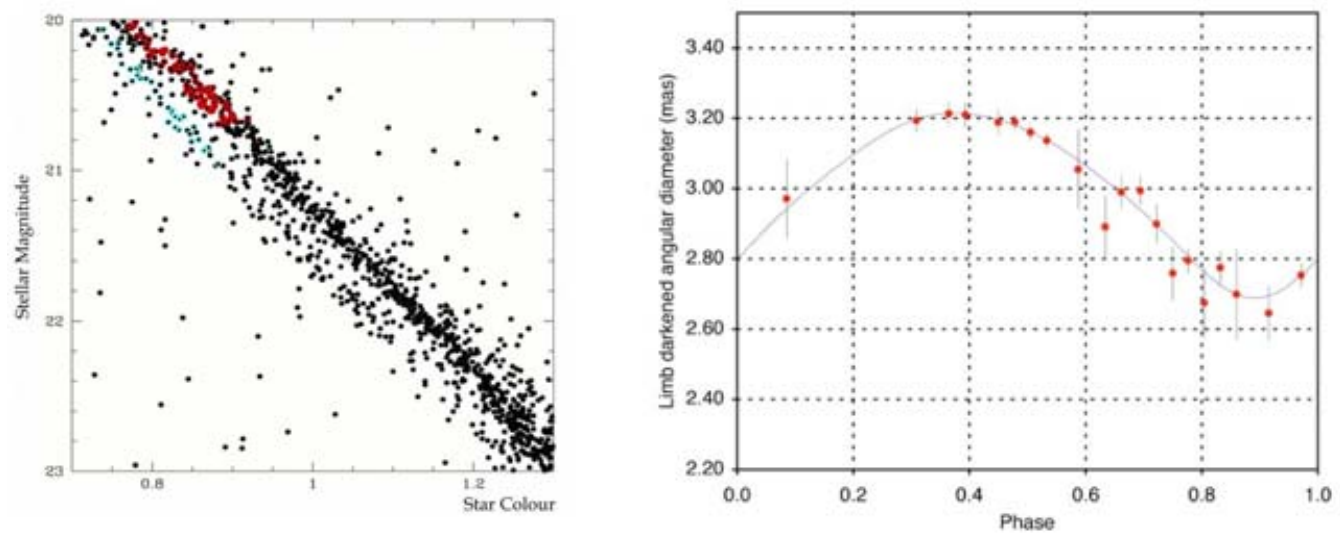

Figure 3. (a) The colour-magnitude diagram for the globular cluster $\omega$-Centauri observed by the VLT using multi-object spectroscopy. The main-sequence is double, the lower sequence having enhanced helium abundance (ESO Press Release 07/05). (b) Periodic variations of the diameter of the Cepheid variable L Carinae have been measured by VLT interferometry, resulting in an improved determination of the Cepheid distance scale (ESO Press Release 25/04).

these observations refer to the average features observed in large samples of galaxies, spectra for individual galaxies have been obtained by the Keck and VLT Telescopes which display similar absorption features out to just as large redshifts. The results to be discussed by McCarthy and Bergeron show just how rapidly studies of the stellar populations in extremely distant galaxies have developed since the commissioning of the present generation of 8-10 metre telescopes.

\subsection{Intergalactic medium and large scale structures}

The Keck-1 telescope did a remarkable job in demonstrating the extraordinary power of a 10-metres telescope for the observation of absorption features in the Lyman- $\alpha$ forest of large redshift quasars. The sensitivities were such that neutral hydrogen column densities as low as $10^{12}$ atoms $\mathrm{cm}^{-2}$ could be detected and this enabled estimates of the initial power-spectrum of the perturbations from which low mass systems formed to be determined. In the synthesis of all the data on the form of the initial power-spectrum of galaxies presented by Tegmark and his colleagues, these data are consistent with the standard Harrison-Zeldovich spectrum with $n=1$, modified according to physics of the standard adiabatic $\Lambda$ CDM model (Tegmark et al. 2004).

A recent spectacular example of the use of these techniques using the Keck telescopes has been the observation of the redshifted ultraviolet spectra of very large redshift quasars which may at last have discovered the Gunn-Peterson trough due to diffuse intergalactic neutral hydrogen (Becker et al. 2001) (Figure 5(a)). This has been one of the major goals of astrophysical cosmology for many years. The amount of diffuse neutral hydrogen needed to extinguish the ultraviolet continuum emission of the quasar is not large, but the important point is that diffuse neutral hydrogen has been detected and this is interpreted as the end of the crucial epochs when the reheating and reionisation of the diffuse intergalactic gas took place.

These observations bear upon the issue of the physics of the reheating and ionisation of the primordial intergalactic gas. Constraints on the duration and redshift of this phase have been provided by the Thomson optical depth of the Universe as determined by the Wilkinson MAP experiment. The issues are intimately related to the astrophysics 

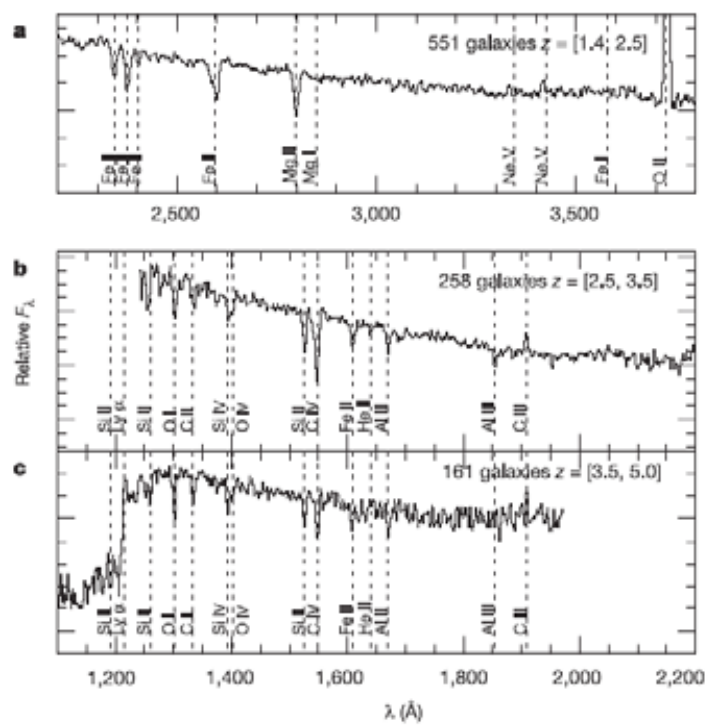

Figure 4. Average spectra of galaxies observed in different redshift ranges as observed by the multi-object spectrograph VIMOS on the VLT. Note the wide range of absorption line features observed even at the largest redshifts (Le Fèvre et al. 2005).

of the first generation of stars and how the process of reionisation and heating actually took place. These problems are also central to the science to be addressed by the Square Kilometre Array (SKA) and so there is an important symbiosis between the interests of the radio and optical astronomers.

Another area in which the Keck-1 telescope made ground-breaking observations during the 1990s was the detection of distant star-forming galaxies by searching for ultraviolet drop-out galaxies. Steidel's surveys of these Lyman-break galaxies enabled evidence on the evolutionary status and spatial distribution of these galaxies to be determined (Steidel et al. 1998). The extension of this technique to even greater redshifts by observing the deficit of redshifted ultraviolet photons has been extended to longer and longer wavelengths resulting in some of the most distant galaxies known, certainly up to redshifts of 6 and possibly beyond (Figure $5(\mathrm{~b})$ ).

\subsection{Galaxy formation-evolution and cosmology}

This discussion leads naturally to the issues of galaxy formation, cosmology and the evolution of galaxies. Again, the Keck-1 telescope got off to a very fast start in making spectroscopic surveys of faint galaxies in the 1990s, the limiting magnitude to which spectra could be obtained being about 24. This was a somewhat tantalising magnitude limit since it is at about this magnitude that the excess of faint blue galaxies really begins to take off. The pioneering surveys of Cowie and his colleagues showed that redshifts up to about 1.7 could be observed for these magnitude-limited samples and that there was evidence for evolution of the numbers of large redshift galaxies (Cowie et al. 1996).

Since these observations of the mid-1990s, the use of multi-object spectroscopy with the VLT and with Subaru have demonstrated how very much larger samples can now be studied. For example, LeFèvre and his colleagues have used the CFHT and the VIMOS instrument on the VLT to study a total sample of about 8,000 galaxies selected in red light. It has turned out that almost 1,000 of these are bright, vigorously star-forming 

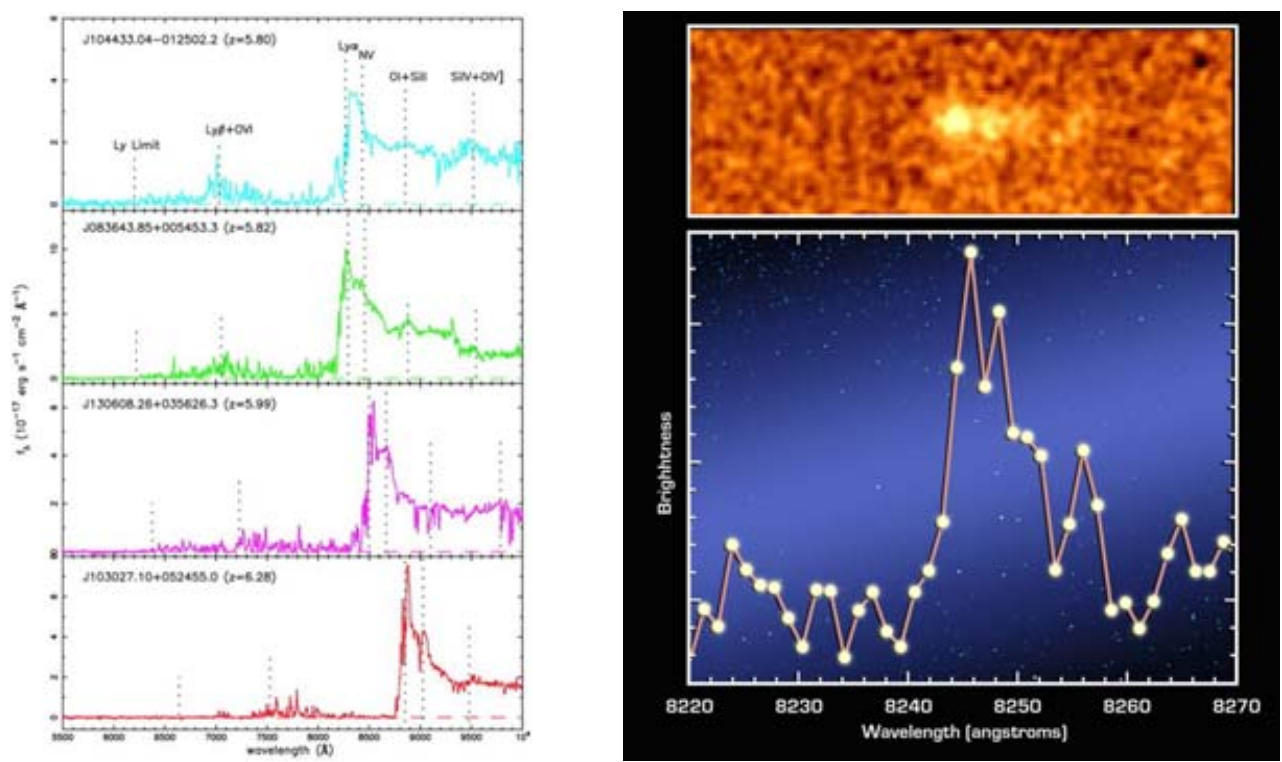

Figure 5. (a) The spectra of four very large redshift quasars, all of which show strong absorption features to the short wavelength side of the redshifted Lyman- $\alpha$ line. In the largest redshift quasar (bottom spectrum), the continuum intensity drops to zero, indicating the presence of diffuse intergalactic neutral hydrogen (Becker et al. 2001). (b) The spectrum of a very red galaxy observed in the Chandra Deep Field South was obtained by Keck Telescope using the Deimos spectrograph. The galaxy has redshift $z=6$ (Keck Observatory Press Release April 2005).

galaxies which must have been formed between 9 and 12 billion years ago (Le Fèvre et al. 2005).

The push to yet larger redshifts has continued, a good example being the very red object found in the Chandra Deep Field South. The spectrum of this object shows a strong asymmetric line which is interpreted as the Lyman- $\alpha$ line, redshifted to the far red end of the optical waveband (Figure 5(b)). A number of these Lyman- $\alpha$ galaxies have now been detected out to redshifts of 6 . Gravitationally lensed objects have also been used to discover galaxies at even larger redshifts. At the moment these are mostly photometric redshifts, but they are indicative that star-forming galaxies are present at these very large redshifts and they must be among the earliest galaxies to have formed.

The large telescopes have also had a key role to play in the various large redshift supernova projects which seek to define with some precision the value of the dark energy density parameter and, potentially, how it might change with redshift.

A further example of the types of cosmological programmes which are ideally matched to the capabilities of the 8-10 metres class telescopes are the precision absorption line spectra needed to be able to set limits to the variability of the fine-structure constant. For example, in a recent set of observations made with the VLT KUEYEN telescope and the UVES spectrograph, 50 absorption systems were studied along 18 distant quasars lines of sight. The spectra of the quasars were recorded over a total of 34 nights. These figures give some impression of the amount of effort needed to obtain a convincing result with the largest telescopes available in some of the most important cosmological programmes.

In the area of active galaxies, one of the most remarkable results obtained by both the Keck and VLT telescopes has been the measurement of the orbits of infrared stars about the central black hole in our Galaxy. The observations by Ghez and her colleagues at the 

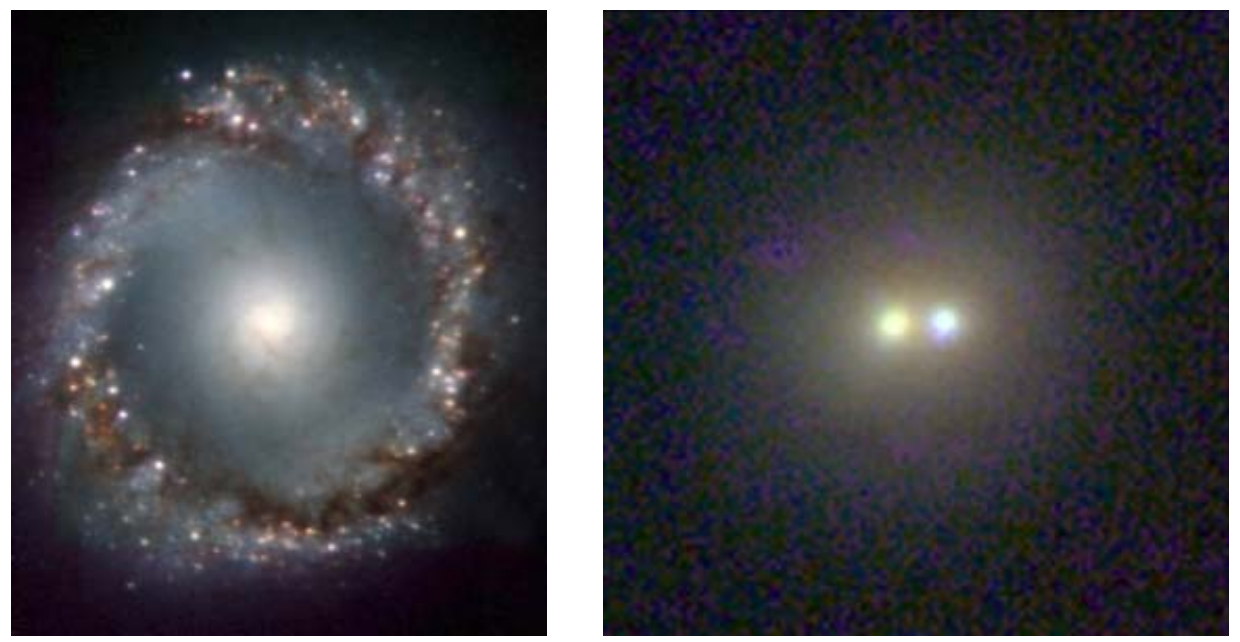

Figure 6. (a) This image of the central regions of NGC1097 was made using the NACO adaptive facility on the VLT. The width of the image is 24 arcsec. More than 300 star forming regions are found in the ring (ESO Press Photo 33/05). (b) This image of a double nucleus of an active galaxy was obtained with the Keck Telescope using an artificial laser guide star (Melbourne et al. 2005). The nuclei are separated by 0.3 arcsec.

Keck Telescopes and Genzel and his colleagues with the VLT have both used adaptive optics to obtain precise orbits for a number of infrared stars (Ghez et al. 2000, Genzel et al. 2002). These observations have shown beyond any doubt that there is a black hole in the Galactic Centre with mass about $3 \times 10^{6} M_{\odot}$. It is significant that both sets of observations had to use various forms of adaptive optics to determine the orbits precisely. This high angular resolution is necessary to demonstrate beyond doubt the presence of a black hole in the Galactic Centre.

Adaptive optics has become a common-user facility for many programmes involving active galactic nuclei. A lovely example is the recent image of NGC 1097 which shows a ring of star-formation about its active nucleus (Figure 6(a)). Equally impressive is the image of the double nucleus of a galaxy obtained by the Keck Telescope using an artificial laser guide star (Figure 6(b)). Observations using this type of adaptive optics system are planned to be an integral part of the next generation of large telescopes.

\subsection{Conclusion}

From this brief summary of some of the demonstrated capabilities of the 8-10 metre telescopes we learn:

Lesson 2 The 8-10 metres class telescopes are more than fulfilling their promise. There are many years of important and exciting observations ahead. Everything needs to be substantiated by independent observations. The value of having the VLT, Gemini, SUBARU and the other large telescopes is that false dogma can be extirpated.

\section{The new expectations of the astronomers}

The result of the success of the 8-10 metres telescopes has been to change the expectations of astronomers for optical-infrared observations.

- Data of superb quality as the normal expectation of large telescope facilities;

- Images of angular resolution $\theta \leqslant 0.4 \operatorname{arcsec}$ as standard; 
Table 1. Illustrating the 'Innovation factor' involved in the construction of the present generation of 8-10 metre telescopes (Courtesy of Dr. Matt Mountain).

\begin{tabular}{lcc}
\hline & $4 \mathrm{~m}(\mathrm{KPNO})$ & $8 \mathrm{~m}(\mathrm{Gemini})$ \\
\hline Cost $(2000)$ & $\$ 47 \mathrm{M}$ & $\begin{array}{c}\text { Scaled cost } \$ 305 \mathrm{M} \\
\text { Actual cost } \$ 100 \mathrm{M}\end{array}$ \\
Innovation factor & $\sim 3$ \\
Image quality & 1 arcsec at $2 \mu \mathrm{m}$ & 0.1 arcsec at $2 \mu \mathrm{m}$ \\
& assuming adaptive optics. \\
\hline
\end{tabular}

- Images with $\theta \sim 0.1$ arcsec with adaptive optics;

- Astronomy with 10-metres telescopes as 'Big Science'.

Thus, the ground has been well prepared for the next generation of Very Large Telescopes, but the expectations are really demanding. Let me give my personal perspective on the proposals for 30 and 100 metre optical-infrared telescopes. I strongly welcome the initiatives to prepare for the next major increase in capability for large telescopes, but, not at any cost! There are a number of reasons for this.

- We need to maintain a broad programme of observational astronomy, not just in optical-infrared astronomy, but over the whole of the electromagnetic spectrum.

- We need to ensure the continuation of the diversity of optical-infrared telescopes. In particular, we need to ensure the vitality of the present generation of 8-10 metres telescopes over the long term. We all know that funding agencies have a tendency to ask what we are to close down in order to fund the operations of new telescope facilities.

- We also know that there is an upper limit to the costs of facilities which national agencies are prepared to fund. All of us remember the case of the Supercolliding Superconductor. In practical terms, we know that the limit for any ground-based telescope facility is $\sim 1-2 \mathrm{~B} \$$ and this has been recognised by the target budgets set for the US 30-metres telescopes and the ESA Overwhelming Large Telescope (OWL), which might be up to 100-metres in diameter.

\section{Scaling relations for the costs of Extremely Large Telescopes}

This is a controversial subject, but it is one which has to be really seriously addressed by the proponents of large optical-infrared telescopes. For the classical design of telescopes before the 8-10 metre era, a reasonable cost-diameter relation was Cost $\propto D^{2.7}$. The great achievement of the current generation of 8-10 metre telescopes has been to break this relation. Many factors have contributed to this, but the largest has come about through the realisation that the cost of computing power has come down so much that it is very much cheaper to make telescopes stiff electronically rather than by making the structures more massive. Let us illustrate this point by looking at the costs of 4 and 8-metre telescopes summarised in Table 1.

The scaling laws shows that an 8 -metres telescope would have cost over $\$ 300 \mathrm{M}$ using traditional technology, but the many innovations adopted in their designs reduced this by a factor of 3 , much of this gain resulting from the use of computers to stiffen the telescope. In fact, we gained much more than a factor of 3 in terms of science return because these costs include the implementation of adaptive optics and so we have gained a very significantly larger factor in terms of scientific capability for a fixed expenditure. 
Table 2. Necessary innovation factors assuming Cost $\propto D^{2.7}$ (Courtesy of Dr. Matt Mountain).

\begin{tabular}{ccc}
\hline Known Costs & Target Costs & Necessary Innovation Factor \\
\hline HST & JWST $6 \mathrm{~m}$ & 10 \\
$(\$ 4.0 \mathrm{~B}$ at 2005 prices $)$ & $(\$ 4.5 \mathrm{~B}$ at 2005 prices $)$ & \\
\hline $\begin{array}{c}\text { Keck }+ \text { LGS AO } \\
(\$ 100 \mathrm{M} \text { at } 2000 \text { prices })\end{array}$ & Thirty Metre Telescope $(\$ 700 \mathrm{M})$ & 4 \\
$\begin{array}{c}\text { Gemini }+ \text { MCAO } \\
(\$ 100 \mathrm{M} \text { at } 2005 \text { prices })\end{array}$ & & 61 \\
\hline $\operatorname{VLT}(\$ 100 \mathrm{~m}$ at 2005 prices $)$ & OWL $100 \mathrm{~m}(\$ 1.5 \mathrm{~B})$ & \\
\hline
\end{tabular}

The concern I have is that we have now played this card and so, going from 8-10 metres upwards, we should be back to the Cost $\propto D^{2.7}$ relation. This is the real concern - where do we find the extra innovation factors necessary to make extremely large telescopes affordable?

As an example, let us work out the innovation factors necessary to make some of the next generation telescopes affordable. This is illustrated in Table 2. It is assumed that all telescopes are diffraction limited at 2 microns. In the case of the HST/JWST comparison, we know that much of the innovation factor comes from the fact that the JWST will be optimised for $2 \mu \mathrm{m}$ and longer wavelengths. A further concern is the scaling law for multiply-conjugated adaptive optics which may scale as a high power of the diameter of the telescope. Number of sub-apertures increases as $D^{2}$ but the amount of computation may go as a much higher power.

Lesson 3 The challenge of going to 30-metres diameter is as great as that of going from the classical generation of optical telescopes to the present generation of 8-10 metres telescopes. The challenge of going to even greater apertures increases dramatically with increasing size.

\section{The hearts and minds of astronomers}

I like to think of the capabilities of the next generation of large telescopes in terms of three levels of sophistication.

(1) Absolutely guaranteed performance: 0.4 arcsec images with active optics, tip-tilt and focus corrections.

(2) Narrow field adaptive optics: diffraction-limited images using single laser guidestars.

(3) Multiply conjugated adaptive optics: wide-field diffraction-limited imaging with multiple laser guide-stars

We need to compare this with what the astronomers really want. This is a topic which I believe really needs to be addressed by the astronomical community and the funding agencies. My impression is that for many astronomers, for whom the sheer collecting power of the telescope is the important factor, level (1) is what they require.

Level (2) is now being offered by the 8-10 metres telescopes and is producing excellent science. Does narrow field adaptive optics produce photometric data? It is encouraging that this is now beginning to be achieved for the photometry of point objects. For example, using classical adaptive optics with a natural guide-star 5 arcsec from the field centre in the $\mathrm{J}$ waveband, the Altair instrument on Gemini has achieved Strehl ratios of 
$53 \%$ and photometric accuracies of $2.5 \%$ for $\mathrm{K} \leqslant 14$. By oversampling the images, even larger Strehl ratios can be achieved. However, we really need more than this. Many of us will want to be assured that we can carry out quantitative photometry of extended objects, such as galaxies at large redshifts, and we really need to know how well this can be achieved.

In level (3), the goal is to achieve wide-field diffraction limited performance. This is the ultimate goal of the various adaptive optics programmes, but it is a real challenge since it involves the use of multiple laser guide stars to map the irregularities at a wide range of altitudes above the telescope. I am sure we will hear a lot about what is possible during this meeting.

It is clear that the use of adaptive optics is beginning to make a significant scientific impact, but we really need to know how essential it is for the typical observing programme. Specifically, how many astronomers use adaptive optics as the normal part of their observing proposals, who are not the people who built the systems or who are not proponents of adaptive optics? How many astronomers need wide-field as opposed to narrow-field adaptive optics and are they prepared to pay for it? I have a suspicion that this may be a generational question and that the newest generation of young astronomers will take to adaptive optics like ducks to water.

Lesson 4 My guesses to the questions are as follows. All astronomers will be very happy with level (a). Many astronomers will be very happy with level (b). If level (c) can be achieved without outrageous cost, astronomers will be supportive, but it has yet to be demonstrated that this can be done.

My final thoughts are that, in many ways, the scientific case for extremely large telescope is the easy bit and I am certain we will hear many wonderful, creative ideas during the course of this symposium. I am sure we also need equally imaginative break-throughs in telescope design and construction. I am optimistic that there are already the people in the audience who will come up with these and achieve even better science than we can imagine.

\section{References}

Becker, R.H., Fan, X., and 29 authors 2001, AJ 122, 2850

Cowie, L.L., Songaila, A., Hu, E.M. \& Cohen, J.D. 1996, AJ 112, 839

Ghez, A.M., Morris, M., Becklin, E.E., Tanner, A. \& Krememek, T. 2000, Nature 407, 349

Le Fèvre, O., Paltani, S. and 47 authors 2005, Nature 437, 519

Melbourne, J., Wright, S.A. and 16 authors 2005, ApJ 625, L27

Schödel, R., Ott, T. and 21 authors 2002, Nature 419, 694

Steidel, C.C. 1998, in: A.V. Olinto, J.A. Frieman \& D.N. Schramm (eds) Eighteenth Texas Symposium on Relativistic Astrophysics and Cosmology, River Edge, N.J.: World Scientific Publishing Company p. 124

Tegmark, M., Strauss, M.A., and 66 authors 2004, Phys. Rev. D 69, 103501 (1-28)

\section{Discussion}

Monnet: Are you not mixing 2 different aspects of the adaptive optics acceptance by the astronomical community: 1) A technical one which comes from the present limitations in sky coverage and wavelength domain, and 2) A scientific one which comes from the fact that natural seeing more or less coincides with the optimum sampling for faint objects with an 8-10m class telescope, but AO is needed for any ELT- class collector? 
LONGAIR: I think there is no contradiction. I am adopting the position that with active optics, tip-tilt and focus corrections, telescopes larger than 10 meter will still have a 'natural seeing' of about 0.4 arcsec. The experts need to confirm, or otherwise, if this is really a correct assumption. If it were correct, many astronomers would be very happy. It may, however, be that even to achieve this with ELTs adaptive optics is required (see the remark by Ellerbroek).

ELLERBROEK: A technical comment: Laser guidestar adaptive optics on ELTs will require multiple guidestars for even narrow field compensated imaging. 8-10 m telescopes are approximately the limit for laser guidestar systems using a single beacon (due to the cone effect).

LONGAIR: Thank you for this clarification. 